\title{
Decentralized Access Control with Anonymous Authentication of Data Stored in Clouds
}

\author{
Mrs. B. Sathyabama" ${ }^{*}$, C. SureshKumar ${ }^{2}$, K. Kesau³ ${ }^{3}$, R. Karthikeyan ${ }^{4}$ \\ ${ }^{1}$ Assistant Professor, PG and Research Department of Computer Applications, Hindusthan College of Arts and \\ Science, Coimbatore, Tamil Nadu, India \\ 2,3,4 PG Scholar, PG and Research Department of Computer Applications, Hindusthan College of Arts and \\ Science, Coimbatore, Tamil Nadu, India
}

\begin{abstract}
The paper proposes a new decentralized access control scheme for secure data storage in clouds that supports anonymous authentication. In the proposed scheme, the cloud verifies the authenticity of the series without knowing the user's identity before storing data. Our scheme also has the added feature of access control in which only valid users are able to decrypt the stored information. The scheme prevents replay attacks and supports creation, modification, and reading data stored in the cloud. We also address user revocation. Moreover, our authentication and access control scheme are decentralized and robust, unlike other access control schemes designed for clouds which are centralized. The communication, computation, and storage overheads are comparable to centralized approaches.
\end{abstract}

Keywords : Dependable Cloud Storage, Decentralized Access Control Scheme.

\section{INTRODUCTION}

Research in cloud computing is receiving a lot of attention from both academic and industrial worlds. In cloud computing, users can outsource their computation and storage to servers (also called clouds) using Internet. This frees users from the hassles of maintaining resources on-site. Clouds can provide several types of services like applications (e.g., Google Apps, Microsoft online), infrastructures (e.g., Amazon's EC2, Eucalyptus, Nimbus), and platforms to help developers write applications (e.g., Amazon's S3, Windows Azure).

Much of the data stored in clouds is highly sensitive, for example, medical records and social networks. Security and privacy are thus very important issues in cloud computing. In one hand, the user should authenticate itself before initiating any transaction, and on the other hand, it must be ensured that the cloud does not tamper with the data that is outsourced. User privacy is also required so that the cloud or other users do not know the identity of the user. The cloud can hold the user accountable for the data it outsources, and likewise, the cloud is itself accountable for the services it provides. The validity of the user who stores the data is also verified. Apart from the technical solutions to ensure security and privacy, there is also a need for law enforcement.

Recently, Wang et al. [2] addressed secure and dependable cloud storage. Cloud servers prone to Byzantine failure, where a storage server can fail in arbitrary ways [2]. The cloud is also prone to data modification and server colluding attacks. In server colluding attack, the adversary can compromise storage servers, so that it can modify data files as long as they are internally consistent. To provide secure 
data storage, the data needs to be encrypted. However, the data is often modified and this dynamic property needs to be taken into account while designing efficient secure storage techniques.

Efficient search on encrypted data is also an important concern in clouds. The clouds should not know the query but should be able to return the records that satisfy the query. This is achieved by means of searchable encryption [3], [4]. The keywords are sent to the cloud encrypted, and the cloud returns the result without knowing the actual keyword for the search. The problem here is that the data records should have keywords associated with them to enable the search. The correct records are returned only when searched with the exact keywords.

Security and privacy protection in clouds are being explored by many researchers. Wang et al. [2] addressed storage security using Reed-Solomon erasure-correcting codes. Authentication of users using public key cryptographic techniques has been studied in [5]. Many homomorphic encryption techniques have been suggested [6], [7] to ensure that the cloud is not able to read the data while performing computations on them. Using homomorphic encryption, the cloud receives ciphertext of the data and performs computations on the ciphertext and returns the encoded value of the result. The user is able to decode the result, but the cloud does not know what data it has operated on. In such circumstances, it must be possible for the user to verify that the cloud returns correct results.

Accountability of clouds is a very challenging task and involves technical issues and law enforcement. Neither clouds nor users should deny any operations performed or requested. It is important to have log of the transactions performed; however, it is an important concern to decide how much information to keep in the log. Accountability has been addressed in TrustCloud [8]. Secure provenance has been studied in [9].

Considering the following situation: A Law student, Alice, wants to send a series of reports about some malpractices by authorities of University $\mathrm{X}$ to all the professors of University $\mathrm{X}$, Research chairs of universities in the country, and students belonging to Law department in all universities in the province. She wants to remain anonymous while publishing all evidence of malpractice. She stores the information in the cloud. Access control is important in such case, so that only authorized users can access the data. It is also important to verify that the information comes from a reliable source. The problems of access control, authentication, and privacy protection should be solved IEEE TRANSACTIONS ON PARALLEL AND DISTRIBUTED SYSTEMS VOL:25 NO:2 YEAR 2014 2 simultaneously. We address this problem in its entirety in this paper.

Access control in clouds is gaining attention because it is important that only authorized users have access to valid service. A huge amount of information is being stored in the cloud, and much of this is sensitive information. Care should be taken to ensure access control of this sensitive information which can often be related to health, important documents (as in Google Docs or Dropbox) or even personal information (as in social networking). There are broadly three types of access control: User Based Access Control (UBAC), Role Based Access Control (RBAC), and Attribute Based Access Control (ABAC). In UBAC, the access control list (ACL) contains the list of users who are authorized to access data. This is not feasible in clouds where there are many users. In RBAC (introduced by [10]), users are classified based on their individual roles. Data can be accessed by 
users who have matching roles. The roles are defined by the system. For example, only faculty members and senior secretaries might have access to data but not the junior secretaries. ABAC is more extended in scope, in which users are given attributes, and the data has attached access policy. Only users with valid set of attributes, satisfying the access policy, can access the data. For instance, in the above example certain records might be accessible by faculty members with more than 10 years of research experience or by senior secretaries with more than 8 years experience. The pros and cons of RBAC and $A B A C$ are discussed in [11]. There has been some work on ABAC in clouds (for example, [12], [13], [14], [15], [16]). All these work use a cryptographic primitive known as Attribute Based Encryption (ABE). The The eXtensible Access Control Markup Language (XACML) [17] has been proposed for ABAC in clouds [18].

An area where access control is widely being used is health care. Clouds are being used to store sensitive information about patients to enable access to medical professionals, hospital staff, researchers, and policy makers. It is important to control the access of data so that only authorized users can access the data. Using $\mathrm{ABE}$, the records are encrypted under some access policy and stored in the cloud. Users are given sets of attributes and corresponding keys. Only when the users have matching set of attributes, can they decrypt the information stored in the cloud. Access control in health care has been studied in [12], [13].

Access control is also gaining importance in online social networking where users (members) store their personal information, pictures, videos and share them with selected groups of users or communities they belong to. Access control in online social networking has been studied in [19]. Such data are being stored in clouds. It is very important that only the authorized users are given access to those information. A similar situation arises when data is stored in clouds, for example in Dropbox, and shared with certain groups of people.

It is just not enough to store the contents securely in the cloud but it might also be necessary to ensure anonymity of the user. For example, a user would like to store some sensitive information but does not want to be recognized. The user might want to post a comment on an article, but does not want his/her identity to be disclosed. However, the user should be able to prove to the other users that he/she is a valid user who stored the information without revealing the identity. There are cryptographic protocols like ring signatures [20], mesh signatures [21], group signatures [22], which can be used in these situations. Ring signature is not a feasible option for clouds where there are a large number of users. Group signatures assume the pre-existence of a group which might not be possible in clouds. Mesh signatures do not ensure if the message is from a single user or many users colluding together. For these reasons, a new protocol known as Attribute Based Signature (ABS) has been applied. ABS was proposed by Maji et al. [23]. In ABS, users have a claim predicate associated with a message. The claim predicate helps to identify the user as an authorized one, without revealing its identity. Other users or the cloud can verify the user and the validity of the message stored. ABS can be combined with $\mathrm{ABE}$ to achieve authenticated access control without disclosing the identity of the user to the cloud.

\section{IMPLEMENTATION}

\section{A. Modules Description}

\section{i. Encryption / Decryption}

We used RSA algorithm for encryption/Decryption. This algorithm is the proven mechanism for secure 
transaction. Here we are using the RSA algorithm with key size of 2048 bits. The keys are split up and stored in four different places. If a user wants to access the file he/she may need to provide the four set of data to produce the single private key to manage encryption/decryption.

\section{B. File Upload / Download}

\section{File Upload}

The client made request to the key manager for the public key, which will be generated according to the policy associated with the file. Different policies for files, public key also differs. But for same public key for same policy will be generated. Then the client generates a private key by combining the username, password and security credentials. Then the file is encrypted with the public key and private key and forwarded to the cloud.

\section{File Download}

The client can download the file after completion of the authentication process. As the public key maintained by the key manager, the client request the key manager for public key. The authenticated client can get the public key. Then the client can decrypt the file with the public key and the private key. The user's credentials were stored in the client itself. During download the file the cloud will authenticate the user whether the user is valid to download the file. But the cloud doesn't have any attributes or the details of the user.

\section{Policy Revocation for File Assured Deletion}

The policy of a file may be revoked under the request by the client, when expiring the time period of the contract or completely move the files from one cloud to another cloud environment. When any of the above criteria exists the policy will be revoked and the key manager will completely removes the public key of the associated file. So no one recover the control key of a revoked file in future. For this reason we can say the file is assuredly deleted. Automatic file revocation scheme is also introduced to revoke the file from the cloud when the file reaches the expiry and the client didn't renew the files duration.

\section{File Access Control}

Ability to limit and control the access to host systems and applications via communication links. To achieve, access must be identified or authenticated. After achieved the authentication process the users must associate with correct policies with the files. To recover the file, the client must request the key manager to generate the public key. For that the client must be authenticated. The attribute based encryption standard is used for file access which is authenticated via an attribute associated with the file. With file access control the file downloaded from the cloud will be in the format of read only or write supported. Each user has associated with policies for each file. So the right user will access the right file. For making file access the attribute based encryption scheme is utilized.

\section{E. Policy Renewal}

Policy renewal is a tedious process to handle the renewal of the policy of a file stored on the cloud. Here we implement one additional key called as renew key, which is used to renew the policy of the file stored on the cloud. The renew key is stored in the client itself.

\section{STUDY ABOUT THE SYSTEM}

\section{A. Existing System}

- Existing work on access control in cloud are centralized in nature. Except and, all other schemes use ABE. The scheme in uses a symmetric key approach and does not support 
authentication. The schemes do not support authentication as well.

- It provides privacy preserving authenticated access control in cloud. However, the authors take a centralized approach where a single key distribution centre (KDC) distributes secret keys and attributes to all users.

\section{Disadvantages of Existing System}

- The scheme in uses asymmetric key approach and does not support authentication.

- Difficult to maintain because of the large number of users that are supported in a cloud environment.

\section{B. Proposed System}

- We propose a new decentralized access control scheme for secure data storage in clouds that supports anonymous authentication.

- In the proposed scheme, the cloud verifies the authenticity of the series without knowing the user's identity before storing data.

- Our scheme also has the added feature of access control in which only valid users are able to decrypt the stored information.

- The scheme prevents replay attacks and supports creation, modification, and reading data stored in the cloud.

\section{Advantages of Proposed System}

- Distributed access control of data stored in cloud so that only authorized users with valid attributes can access them.

- Authentication of users who store and modify their data on the cloud.

- The identity of the user is protected from the cloud during authentication.

\section{SYSTEM DESIGN}

\section{A. System Architecture}

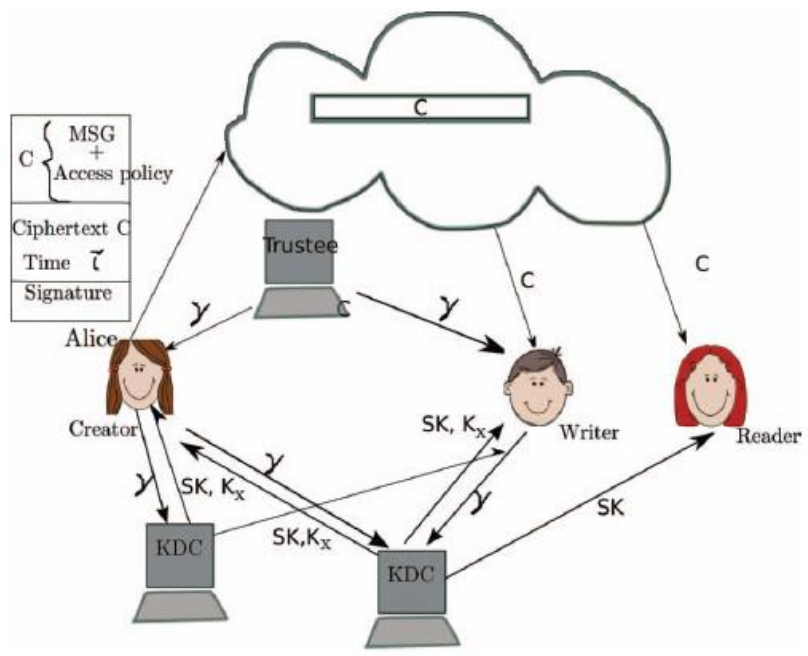

Fig. 4.1 System Architecture

\section{B. Data Flow Diagram}

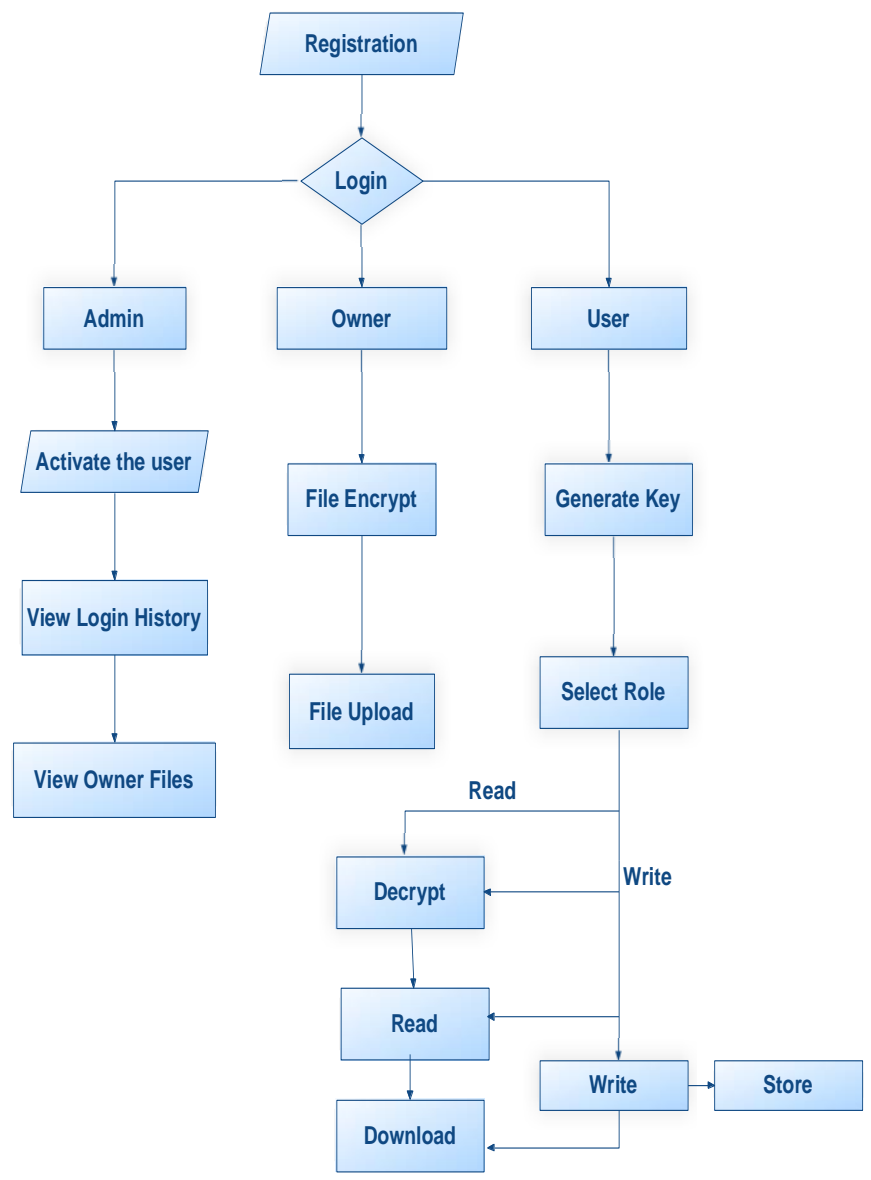

Fig. 4.2 Data Flow Diagram 


\section{Class Diagram}

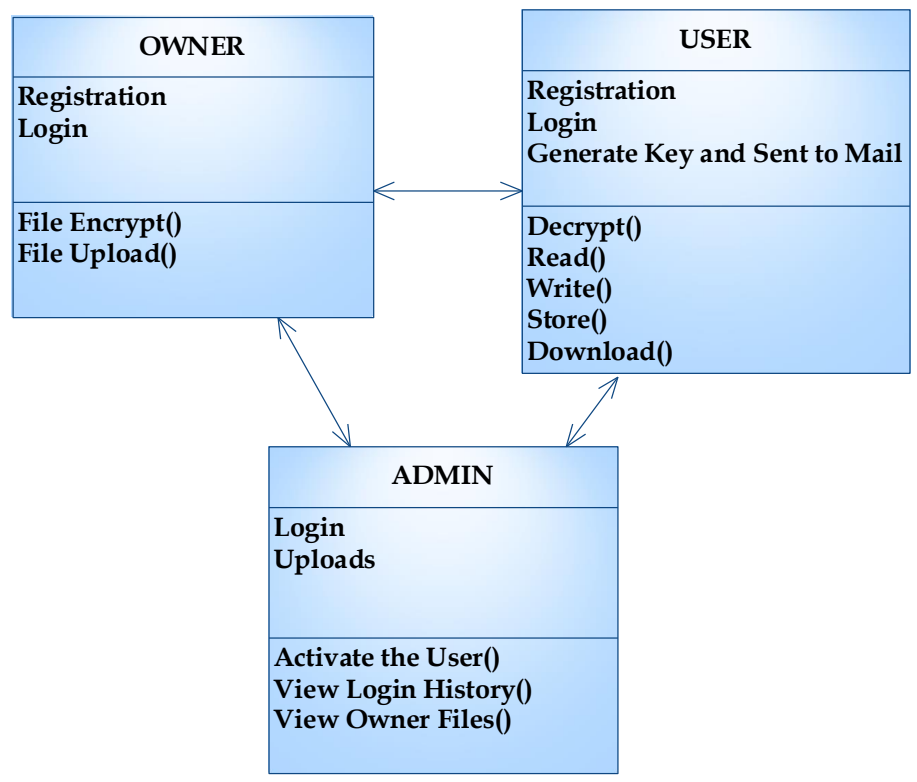

Fig. 4.3 Class Diagram

\section{SAMPLE SCREEN}
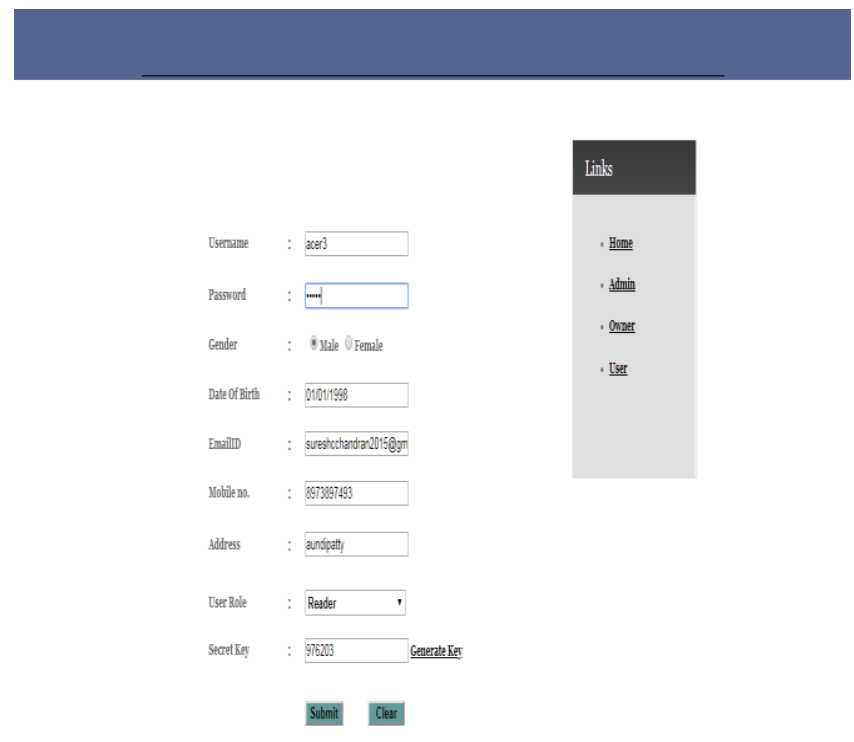

Fig. 5.1 User Registration Page

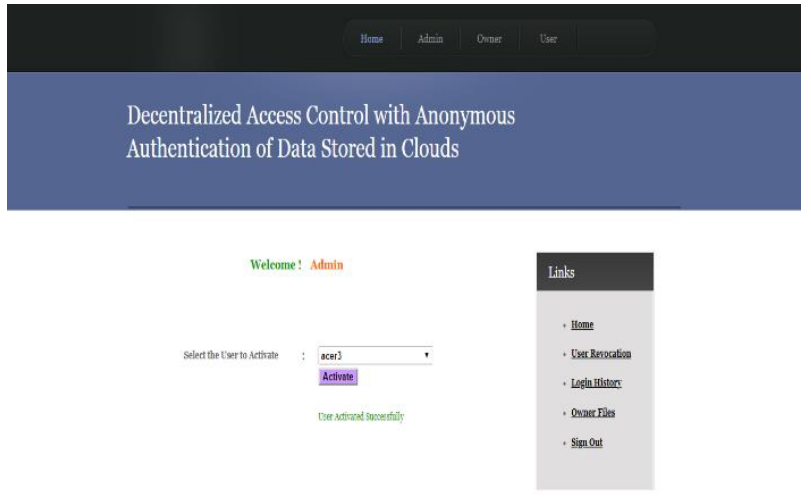

Fig. 5.2 User Activation Page

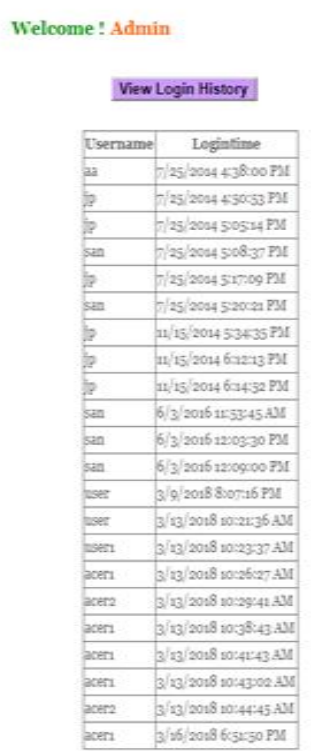

Links

- Home

- Vser Revocation

- Login Histors

- owner Files

- Sign out

Fig. 5.3 History of Logged in Users

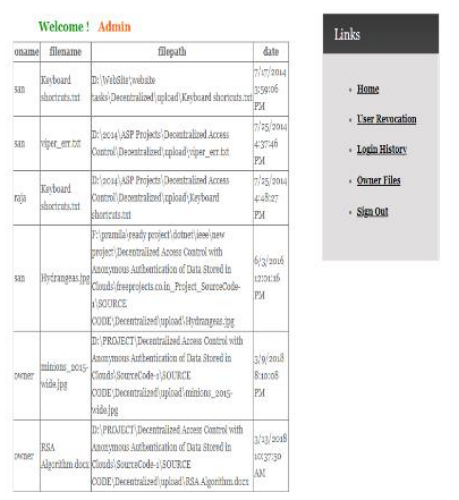

Fig. 5.4 List of Owners for files 


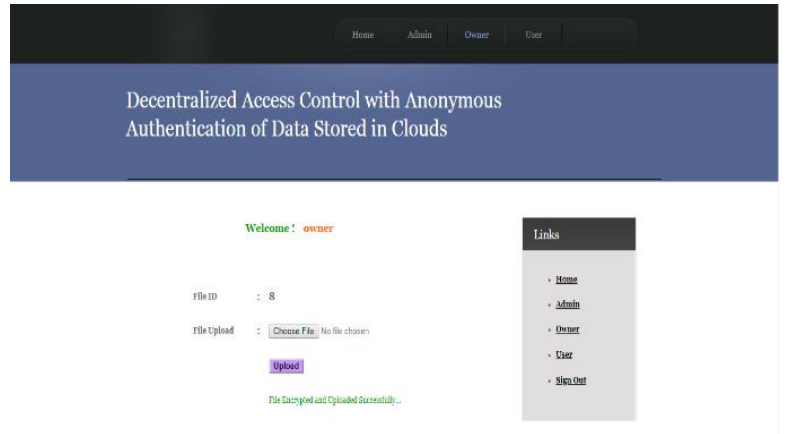

Fig. 5.5 File Upload

Decentralized Access Control with Anonymous

Authentication of Data Stored in Clouds

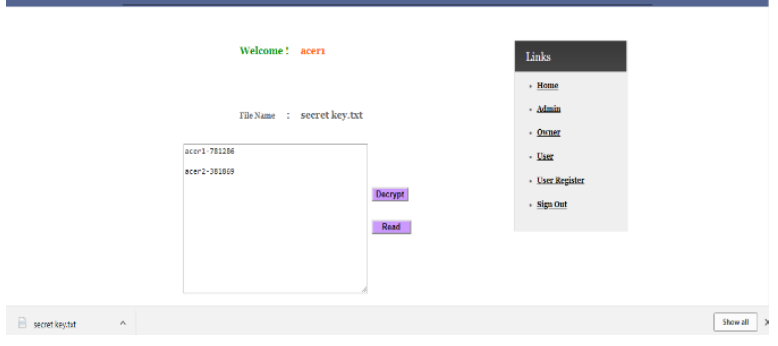

Fig. 5.6 Encrypting and Downloading a File

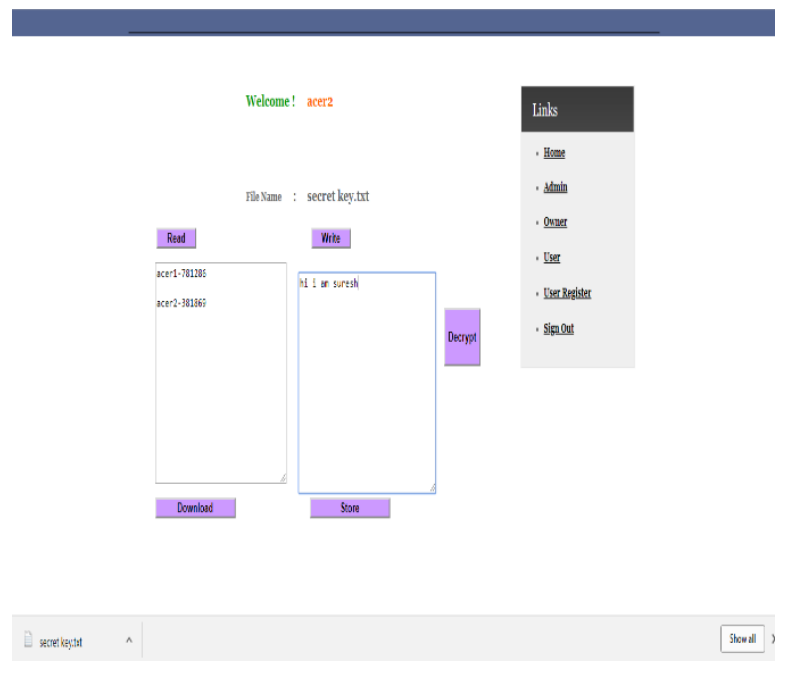

Fig. 5.7 Writing and Downloading a File

\section{CONCLUSION}

We have presented a decentralized access control technique with anonymous authentication, which provides user revocation and prevents replay attacks. The cloud does not know the identity of the user who stores information, but only verifies the user's credentials. Key distribution is done in a decentralized way. One limitation is that the cloud knows the access policy for each record stored in the cloud. In future, we would like to hide the attributes and access policy of a user.

\section{VII.FUTURE ENHANCEMENT}

Decentralized access control technique with anonymous authentication, which provides user revocation and prevents replay attacks. The cloud does not know the identity of the user who stores information, but only verifies the user's credentials. Key distribution is done in a decentralized way. One limitation is that the cloud knows the access policy for each record stored in the cloud. In future, we would like to hide the attributes and access policy of a user. In future the file access policy can be implemented with Multi Authority based Attribute based Encryption. Using the technique it can avoid the number of wrong hits during authentication. Create a random delay for authentication, so the hacker can confuse to identify the algorithm.

\section{REFERENCES}

\section{Journal Papers:}

1. S. Ruj, M. Stojmenovic, and A. Nayak, "Privacy Preserving Access Control with Authentication for Securing Data in Clouds," Proc. IEEE/ACM Int'l Symp. Cluster, Cloud and Grid Computing, pp. 556563, 2012.

2. C. Wang, Q. Wang, K. Ren, N. Cao, and W. Lou, "Toward Secure and Dependable Storage Services in Cloud Computing," IEEE Trans. Services Computing, vol. 5, no. 2, pp. 220-232, Apr.-June 2012.

3. J. Li, Q. Wang, C. Wang, N. Cao, K. Ren, and W. Lou, "Fuzzy Keyword Search Over Encrypted Data 
in Cloud Computing," Proc. IEEE INFOCOM, pp. 441-445, 2010.

4. D.R. Kuhn, E.J. Coyne, and T.R. Weil, "Adding Attributes to Role- Based Access Control," IEEE Computer, vol. 43, no. 6, pp. 79-81, June 2010.

5. H.K. Maji, M. Prabhakaran, and M. Rosulek, "Attribute-Based Signatures: Achieving AttributePrivacy and Collusion-Resistance," IACR Cryptology ePrint Archive, 2008.

6. H.K. Maji, M. Prabhakaran, and M. Rosulek, "Attribute-Based Signatures," Topics in Cryptology - CT-RSA, vol. 6558, pp. 376-392, 2011.

7. K. Yang, X. Jia, and K. Ren, "DAC-MACS: Effective Data Access Control for Multi-Authority Cloud Storage Systems," IACR Cryptology ePrint Archive, p. 419, 2012.

8. A.B. Lewko and B. Waters, "Decentralizing Attribute-Based Encryption,” Proc. Ann. Int'l Conf. Advances in Cryptology (EUROCRYPT), pp. 568588, 2011.

9. J. Hur and D. Kun Noh, "Attribute-Based Access Control with Efficient Revocation in Data Outsourcing Systems," IEEE Trans. Parallel and Distributed Systems, vol. 22, no. 7, pp. 1214-1221, July 2011.

\section{Proceedings Papers:}

1. S. Kamara and K. Lauter, "Cryptographic Cloud Storage," Proc. 14th Int'l Conf. Financial Cryptography and Data Security, pp. 136- 149, 2010.

2. H. Li, Y. Dai, L. Tian, and H. Yang, "Identity-Based Authentication for Cloud Computing," Proc. First Int'l Conf. Cloud Computing (CloudCom), pp. 157166, 2009.

3. A.-R. Sadeghi, T. Schneider, and M. Winandy, "Token-Based Cloud Computing," Proc. Third Int'l Conf. Trust and Trustworthy Computing (TRUST), pp. 417-429, 2010.

4. R. Lu, X. Lin, X. Liang, and X. Shen, "Secure Provenance: The Essential of Bread and Butter of Data Forensics in Cloud Computing," Proc. Fifth
ACM Symp. Information, Computer and Comm. Security (ASIACCS), pp. 282-292, 2010.

5. D.F. Ferraiolo and D.R. Kuhn, "Role-Based Access Controls," Proc. 15th Nat'l Computer Security Conf., 1992.

6. M. Li, S. Yu, K. Ren, and W. Lou, "Securing Personal Health Records in Cloud Computing: Patient-Centric and Fine-Grained Data Access Control in MultiOwner Settings," Proc. Sixth Int'l ICST Conf. Security and Privacy in Comm. Networks (SecureComm), pp. 89-106, 2010.

7. S. Yu, C. Wang, K. Ren, and W. Lou, "Attribute Based Data Sharing with Attribute Revocation," Proc. ACM Symp. Information, Computer and Comm. Security (ASIACCS), pp. 261-270, 2010.

8. G. Wang, Q. Liu, and J. Wu, "Hierarchical AttributeBased Encryption for Fine-Grained Access Control in Cloud Storage Services," Proc. 17th ACM Conf. Computer and Comm. Security (CCS), pp. 735-737, 2010.

9. F. Zhao, T. Nishide, and K. Sakurai, "Realizing FineGrained and Flexible Access Control to Outsourced Data with Attribute-Based Cryptosystems," Proc. Seventh Int'l Conf. Information Security Practice and Experience (ISPEC), pp. 83-97, 2011.

10. S. Ruj, A. Nayak, and I. Stojmenovic, "DACC: Distributed Access Control in Clouds," Proc. IEEE 10th Int'l Conf. Trust, Security and Privacy in Computing and Communications (TrustCom), 2011.

11. S. Jahid, P. Mittal, and N. Borisov, "EASiER: Encryption-Based Access Control in Social Networks with Efficient Revocation," Proc. ACM Symp. Information, Computer and Comm. Security (ASIACCS), 2011.

12. R.L. Rivest, A. Shamir, and Y. Tauman, "How to Leak a Secret," Proc. Seventh Int'l Conf. Theory and Application of Cryptology and Information Security (ASIACRYPT), pp. 552-565, 2001.

13. X. Boyen, "Mesh Signatures," Proc. 26th Ann. Int'l Conf. Advances in Cryptology (EUROCRYPT), pp. 210-227, 2007. 
14. D. Chaum and E.V. Heyst, "Group Signatures," Proc. Ann. Int'l Conf. Advances in Cryptology (EUROCRYPT), pp. 257-265, 1991.

15. A. Sahai and B. Waters, "Fuzzy Identity-Based Encryption," Proc. Ann. Int'l Conf. Advances in Cryptology (EUROCRYPT), pp. 457-473, 2005.

16. V. Goyal, O. Pandey, A. Sahai, and B. Waters, "Attribute-Based Encryption for Fine-Grained Access Control of Encrypted Data," Proc. ACM Conf. Computer and Comm. Security, pp. 89-98, 2006.

17. J. Bethencourt, A. Sahai, and B. Waters, "CiphertextPolicy Attribute-Based Encryption," Proc. IEEE Symp. Security and Privacy, pp. 321-334, 2007.

18. X. Liang, Z. Cao, H. Lin, and D. Xing, "Provably Secure and Efficient Bounded Ciphertext Policy Attribute Based Encryption," Proc. ACM Symp. Information, Computer and Comm. Security (ASIACCS), pp 343-352, 2009.

19. M. Chase, "Multi-Authority Attribute Based Encryption," Proc. Fourth Conf. Theory of Cryptography (TCC), pp. 515-534, 2007.

20. H. Lin, Z. Cao, X. Liang, and J. Shao, "Secure Threshold Multi-Authority Attribute Based Encryption without a Central Authority," Proc. Progress in Cryptology Conf. (INDOCRYPT), pp. 426-436, 2008.

21. M. Chase and S.S.M. Chow, "Improving Privacy and Security in Multi-Authority Attribute-Based Encryption," Proc. ACM Conf. Computer and Comm. Security, pp. 121-130, 2009.

22. M. Green, S. Hohenberger, and B. Waters, "Outsourcing the Decryption of ABE Ciphertexts," Proc. USENIX Security Symp., 2011.

23. W. Wang, Z. Li, R. Owens, and B. Bhargava, "Secure and Efficient Access to Outsourced Data," Proc. ACM Cloud Computing Security Workshop (CCSW), 2009.
Thesis:

1. C. Gentry, "A Fully Homomorphic Encryption Scheme," PhD dissertation, Stanford Univ., http://www.crypto.stanford.edu/ craig, 2009.

2. R.K.L. Ko, P. Jagadpramana, M. Mowbray, S. Pearson, M. Kirchberg, Q. Liang, and B.S. Lee, "Trustcloud: A Framework for Accountability and Trust in Cloud Computing," HP Technical Report HPL-2011-38,

http://www.hpl.hp.com/techreports/2011/HPL2011-38.html, 2013.

3. A. Beimel, "Secure Schemes for Secret Sharing and Key Distribution," PhD thesis, Technion, Haifa, 1996.

\section{Web References:}

1. http://docs.oasis-open.org/xacml/3.0/xacml-3.0core-spec-cs-01-en.pdf, 2013.

2. http://securesoftwaredev.com/2012/08/20/xacml-inthe-cloud, 2013.

3. http://crypto.stanford.edu/pbc/, 2013.

4. "Libfenc: The Functional Encryption Library,"http://code. google.com/p/libfenc/, 2013.

\section{Cite this article as :}

Mrs. B. Sathyabama, C. SureshKumar, K. Kesau, R. Karthikeyan, "Decentralized Access Control with Anonymous Authentication of Data Stored in Clouds", International Journal of Scientific Research in Science and Technology (IJSRST), Online ISSN : 2395-602X, Print ISSN : 2395-6011, Volume 6 Issue 5, pp. 34-42, September-October 2019.

Available at doi : https://doi.org/10.32628/IJSRST196476 Journal URL : http://ijsrst.com/IJSRST196476 